\title{
On Cardinality of Fuzzy Sets
}

\author{
Mamoni Dhar \\ Assistant Profess or, Department of Mathematics, Science College, Kokrajhar, Assam, India \\ Email: mamonidhar@rediffmail.com; mamonidhar@gmail.com
}

\begin{abstract}
In this article, we would like to revisit and comment on the widely used definition of cardinality of fuzzy sets. For this purpose we have given a brief description of the history of development of fuzzy cardinality. In the process, we can find that the existing definition fails to give a proper cardinality while dealing with complementation of fuzzy sets. So there arises the need of defining the cardinality in a different manner. Here a new definition of cardinality is proposed which is rooted in the definition of complementation of fuzzy sets on the basis of reference function. This definition of cardinality will inevitably play an important role in any problem area that involves complementation. Further, some important results are proven with the help of the proposed definition and it is found that these properties are somewhat analogus to those obtained with the help of the existing definition.
\end{abstract}

Index Terms - Membership Value, Reference Function, Excluded Middle Laws, Fuzzy Cardinality of Fuzzy Sets

\section{Introduction}

In real world, the complexity generally arises fro $m$ uncertainty in the form of ambiguity. Uncertainty arises due to partial information about a problem, or due to information which is not fully reliable, or due to inherent imprecision in language with which the problem is defined or due to receipt of information from more than one source. Fuzzy set theory is an excellent mathematical tool to handle the uncertainty arising due to vaugeness, Zadeh [1].

A fuzzy set $\mathrm{A}$ is denoted as $\quad\left\{\left(x, \mu_{A}(x)\right), x \in \Omega\right\}$ where $\mu_{A}(x)$ is the grade of membership of $\mathrm{x}$ in $\mathrm{A}$. Here $\quad \mu_{A}(x)$ is a real number satisfying $0 \leq \mu_{A}(x) \leq 1$. The complement of the fuzzy set $\mathrm{A}$ is denoted by $A^{c}$ and is defined by a membership function

$$
\mu_{A}^{c}(x)=1-\mu_{A}(x), \forall x \in \Omega
$$

Since fuzzy set theory proposed by Zadeh, it has been developed in theory and applications in the past 45 years. In fuzzy set theory, we can see the use of the term cardinality which is most commonly used concept in many areas. Cardinality belongs to most important and elementary characteristics of a set. The cardinality of a crisp set is the number of elements in the set. Using fuzzy sets which are many-valued generalization of sets, one likes to have for them analogus characteristics.

The concept of cardinality of fuzzy sets received a lot of attention from theresearchers from the beginning of fuzzy set theory. Since an element can partially belong to a fuzzy set, a natural generalization of the classical notion of cardinality is to weigh each element by its membership degree, which gives us the following definition for cardinality of a fuzzy set. There are several approaches to the cardinality of fuzzy sets. One group of them had constructive approaches. Following these approaches, we get a single number or alternatively a fuzzy set as cardinality of fuzzy set. The first concept of this kind was proposed and discussed in De Luca and Termini [2]. In many applications, one prefers a simple scalar approximation of card inality of a fuzzy set.

Scalar cardinality of a fuzzy set is the sum of the membership values of all elements of the fuzzy set. In particular, scalar cardinalities of a fuzzy set which associate to each fuzzy set a positive real nu mber. In the similar way, fuzzy cardinalities of a fuzzy set which associate to any fuzzy set a convex fuzzy natural number.

The fuzzy cardinality of fuzzy sets is itself also a fuzzy set on the universe of natural numbers. The first definition of fuzzy card inality of fuzzy sets (say A) by means of mapping from the set of natural numbers to the interval $[0,1]$, was proposed by Zadeh [3, 4]. But fuzzy cardinality of fuzzy sets is beyond the scope of this article and for this reason, we would not like to discuss about it in details in this article.

Since an element can partially belong to a fuzzy set, a natural generalization of the classical notion of cardinality is to weigh each element by its membership degree, which resulted in the following formula for cardinality of a fuzzy set:

$$
|A|=\sum \mu_{A}(x), x \in \Omega
$$

This $|A|$ is called the sigma- count of $\mathrm{A}$.

That is to say that the cardinality of a fuzzy set A, the so called sigma- count, is expressed as the sum of the membership values of $\mathrm{A}$. This approach to cardinality 
of fuzzy sets is convenient in applications and therefore favoured by many practioners. However there are many approaches to this evaluation. The problem of counting fuzzy sets has generated a lot of literature since Zadeh's initial conception. It is most widely used concept in fuzzy areas since it is useful in answering many questions. Therefore, it plays an important role in fuzzy databases and information systems. Furthermore, for simplicity and clarity of the matter under consideration, we can name few areas which mainly depend on cardinality, for example, for finding subsethood, similarity and comparison between two fuzzy sets, which are very much in use in fuzzy areas, the concept of cardinality, plays a key role.

Inclusion measure between two fuzzy sets is the degree to which a fuzzy set is a subset of another fuzzy set. After Zadeh, who first gave a definition of inclusion of two fuzzy sets, Kosko extended the inclusion to the subsethood by allowing for degrees of subsethood of one fuzzy set to another. Afterwards more variants and expressions for subsethood for two fuzzy sets were studied. But in finding these relationships the concept of cardinality is used.

On the other hand, similarity measures in many contexts are used as a mathematical tool for expressing quantitatively to what extent two objects or more formally two instances of a given mathematical structure resemble each other. These were originally introduced to express the degree of comparision between two fuzzy sets. Similarity is perhaps the most frequently used most difficult to quantify and most universally employed type of compatability measure. But it is seen in most of the cases, cardinality is used to find similarity measure.

Most of these results which are of considerable significance are thus far from logical backgrounds. There are innumerable cases in which we can see that the results were obtained with the help of cardinality of fuzzy sets, all these cannot be mentioned. Seeing its importance in the theory of fuzzy sets, we would like to say that the cardinality should be defined properly in order to produce a logical result.

Here in this article, our main concern is with defining cardinality of a fuzzy set in a manner which is consistent with the new definition of complementation. It is necessary to define it accordingly because we do not agree with the existing definition of complementation as there are some laggings in that kind of definition. As a consequence of which we tried to put forward a new definition of cardinality for future studies. It is important to mention here that this new proposal does not affect the cardinality of usual fuzzy sets because in the definition of complement of a fuzzy set, fuzzy membership value and the fuzzy membership function have to be different, in the sense that for a usual fuzzy set the membership value and membership function is of course equivalent. It is for this reason, while dealing with complementation, we should be careful about these two; otherwise we would have to be contended with illogical result. In order to avoid any such confusion regarding this, we prefer to write in short about the new definition of complementation of fuzzy sets on the basis of reference function as introduced by Baruah [5, 6, $7 \& 8]$. Except for the brief overview, here we do not like to examine their properties and procedures by which these are manipulated. Their detailed coverage can be found in Baruah [6].

Our definition of cardinality is a result of the motivation from this definition of complementation of fuzzy sets. It is expected that this definition would be of great help in finding an accurate estimate of cardinality of fuzzy sets particularly of complementation

The paper is organized as follows: Section II describes the definition of complementation of fuzzy sets which is used to develop the new definition of cardinality of fuzzy sets. Section III introduces the new definition of cardinality of fuzzy sets. Section IV presents some numerical examples which are in accordance with the new definition of cardinality. Section V cites some numerical examples to show the cases where our definition differs from the existing definition of cardinality of fuzzy sets. Finally, Section VI presents our conclusions.

\section{Baruah's Definition of Complementation of Fuzzy Sets}

Baruah [5, 6, $7 \& 8$ \& has defined a fuzzy number $\mathrm{N}$ with the help of two functions: a fuzzy membership function $\mu_{2}(x)$ and a reference function $\mu_{1}(x)$ such that $0 \leq \mu_{1}(x) \leq \mu_{2}(x) \leq 1$. Then for a fu zzy number denoted by $\left\{x, \mu_{1}(x), \mu_{2}(x), x \in \Omega\right.$ we would call $\left\{\mu_{2}(x)-\mu_{1}(x)\right\}$ as the fuzzy membership value, which is different from fuzzy membership function. As an illustration of the above formalization, we are going to mention the following few lines.

In accordance with the process discussed above, a fuzzy set defined by

$$
A=\{x, \mu(x), x \in \Omega\}
$$

would be defined in this way as

$$
A=\{x, \mu(x), 0, x \in \Omega\}
$$

so that the complement would become

$$
A^{c}=\{x, 1, \mu(x), x \in \Omega\}
$$

It is important to note here that in most cases of practical significance, it is desirable to consider reference function for fuzzy compliments. Fuzzy sets operation like union and intersection are also defined accordingly in order to keep pace with the aforesaid 
definition of complementation. All these results have been already considered and discussed in our previous works as can be found in Dhar [9, 10, 11, 12, 13 \& 14]. So we would not like to mention about all these in details herein.

From the above definition of complementation, we would like to say that the cardinalities of complementation of fuzzy sets obtained by using the existing definitions of complementation would no longer yield satisfactory results. In a subject like mathematics, we should not go ahead with anything which is not logical. That is why we would like to propose a new concept in finding the cardinality of fuzzy sets. The proposed defintion is obtained within a proper mathematical framework and it is defined in accordance with the suggested definition of complementation. The proposed technique has been demonstrated in the in the following section with some examples so as to make the matter clear and simple.

\section{New Definition of Cardinality of Fuzzy Sets}

It is important to mention here that since we would like to define fuzzy sets with the help of two functions such as fuzzy membership function and fuzzy reference function, it is therefore necessary to define cardinality of fuzzy sets accordingly. That is to say that the method we will discuss here for determining cardinality is based on this assumption. In parallel with what had been done for cardinalities of fuzzy sets, we shall define the cardinality of a fuzzy set A as:

$$
|A|=\operatorname{card}(A)=\sum\left\{\mu_{2}(x)-\mu_{1}(x)\right\}, x \in \Omega
$$

Since in case of usual fuzzy sets membership value and membership function indicate the same thing, so for usual fuzzy sets we can proceed with the existing definition. In case of complementation these two differs and hence we stress on this definition of cardinality to avoid any misleading result. So for finding cardinality of complement of a fuzzy set, there is no other way than to go for an alternate definition. Here one thing is worth mentioning that our result is derived in line with scalar card inalities. So it can be said that the cardinality of a fuzzy set defined in the above manner will play a very key role in various fuzzy areas henceforth.

Further, it is important to mention here that Kosko [15] established some propositions for cardinality of usualfuzzy sets which were expressed as follows:

\section{Proposition1}

$$
\text { If } A \subseteq B \text { then } \sum \operatorname{count}(A) \subseteq \sum \operatorname{count}(B)
$$

Proposition 2

$$
\begin{aligned}
& \sum \operatorname{Count}(A)+\sum \operatorname{Count}(B)= \\
& \sum \operatorname{Count}(A \cup B)+\sum \operatorname{Count}(A \cap B)
\end{aligned}
$$

Proposition 3

$\sum \operatorname{Count}(A \cap B) \leq$

$$
\begin{aligned}
& \min \left(\sum \operatorname{count}(A), \sum \operatorname{count}(B)\right) \\
& \leq \min \left(\sum \operatorname{count}(A), \sum \operatorname{count}(B)\right) \\
& \leq \sum \operatorname{count}(A \cup B)
\end{aligned}
$$

Here we claim that these propositions hold for the cardinality of complement of fuzzy sets also, if we proceed with the proposed definition. This would be clear with the help of numerical examples cited below.

\section{Numerical Examples}

In this section we have tried to make the matter clear and complete with the help of some nu merical examples.

Let us consider a fuzzy set in the usualcase as

$$
\mathrm{A}=\{(1,0.2),(2,0.4),(3,0.6),(4,0.8),(5,1)\}
$$

And the complement of the set A, according to the existing definition is

$$
A^{c}=\{(1,0.8),(2,0.6),(3,0.4),(4,0.2),(5,0)\}
$$

Now the cardinality of the fuzzy set $A^{c}$, in accordance with the existing definition would be

$$
\begin{aligned}
\left|A^{c}\right| & =0.8+0.6+0.4+0.2+0 \\
& =2
\end{aligned}
$$

Then the fuzzy set A would take the following form if the new definition of fuzzy sets based on reference function is taken into consideration

$\mathrm{A}=\{(1,0.2,0),(2,0.4,0),(3,0.6,0),(4,0.8,0),(5,1$, $0)\}$,

Where as the complement of this set $A^{c}$ would take the following form

$$
\begin{aligned}
A^{c}= & \{(1,1,0.2),(2,1,0.4),(3,1,0.6),(4,1,0.8),(5, \\
& 1,1)\}
\end{aligned}
$$

According to the proposed definition of cardinality, the cardinality of the fuzzy set $A^{c} \quad$ will be calculated as

$$
\left|A^{c}\right|=(1-0.2)+(1-0.4)+(1-0.6)+(1-0.8)+(1-1)
$$




$$
\begin{aligned}
& =.8+.6+.4+.2+0 \\
& =2
\end{aligned}
$$

The result obtained will coincide with the cardinality of $A^{c}$ if it were calculated by using existing definition. But it would be worth mentioning here that there will be some situations where we shall get results different from those calculated by existing formula especially when it involves operation with complementation.

Now for proving the propositions as proposed by Kosko [15], we would like to cite the following example for illustration purposes.

Let us consider two fuzzy sets

$A=\{(1,0.2),(2,0.5),(3,0.8),(4,1),(5,0.7),(6,0.3)\}$

And

$$
\mathrm{B}=\{(3,0.2),(4,0.4),(5,0.6)\}
$$

This set B can be written in the following form,

$$
\mathrm{B}=\{(1,0),(2,0),(3,0.2),(4,0.4),(5,0.6),(6,0)\}
$$

So that there complements of the fuzzy sets A and B are

$A^{c}=\{(1,1,0.2),(2,1,0.5),(3,1,0.8),(4,1,1),(5,1,0.7)$, $(6,1,0.3)\}$

$B^{c}=\{(1,1,0),(2,1,0),(3,1,0.2),(4,1,0.4),(5,1,0.6)$, $(6,1,0)\}$

Here we can say that me mbership values of $A^{c}$ is less than that of $B^{c}$ and so $A^{c}$ can be considered as a subset of $B^{c}$.

Here, we get

$$
\begin{aligned}
\left|A^{c}\right| & =(1-0.2)+(1-0.8)+(1-1)+(1-0.7)+(1-0.3) \\
& =.8+.5+.2+0+.3+.7 \\
& =2.5 \\
\left|B^{c}\right| & =(1-0)+(1-0)+(1-0.2)+(1-0.4)+(1-0.6)+(1-0) \\
& =1+1+.8+.6+.4+1 \\
& =4.8
\end{aligned}
$$$$
\text { Thus } A^{c} \subseteq B^{c} \text { if then }\left|A^{c}\right| \leq\left|B^{c}\right|
$$

This proves the first proposition.

Here, we see that

$$
\left|A^{c}\right|+\left|B^{c}\right|=2.5+4.8=7.3
$$

Again, we get the following results

$$
\begin{aligned}
& \begin{array}{l}
A^{c} \cup B^{c} \\
=\{(1,1,0),(2,1,0),(3,1,0.2),(4,1,0.4), \\
\quad(5,1,0.6),((6,1,0)\}
\end{array} \\
& A^{c} \cap B^{c} \\
& =\{(1,1,0.2),(2,1,0.5),(3,1,0.8),(4,1,1), \\
& \quad(5,1,0.7),(6,1,0.3)\} \\
& \left|A^{c} \bigcup B^{c}\right|=1+1+0.8+0.6+0.4+1=4.8 \\
& \left|A^{c} \cap B^{c}\right|=0.8+0.5+0.2+0+0.3+0.7=2.5
\end{aligned}
$$

Here, we get

$$
\left|A^{c} \cap B^{c}\right|+\left|A^{c} \cup B^{c}\right|=7.3
$$

Hence the following result is obtained

$$
\left|A^{c}\right|+\left|B^{c}\right|=\left|A^{c} \cap B^{c}\right|+\left|A^{c} \cup B^{c}\right|
$$

This proves the second proposition.

Lastly, we have,

$\min \left(\left|A^{c}\right|,\left|B^{c}\right|\right)=\min (2.5,4.8)=2.5$

and

$\max \left(\left|A^{c}\right|,\left|B^{c}\right|\right)=\max (2.5,4.8)=4.8$

$\left|A^{c}\right| \leq \min \left(\left|A^{c}\right|,\left|B^{c}\right|\right) \leq \max \left(\left|A^{c}\right|,\left|B^{c}\right|\right) \leq\left|B^{c}\right|(13)$

which is the third proposition.

From the above, we can say that the new method fulfills the concept of subadditivity. That can be expressed $A^{c}$ symbolically as follows:

$$
\left|A^{c} \cup B^{c}\right| \leq\left|A^{c}\right|+\left|B^{c}\right|
$$

Thus we can say that the three propositions which hold for usual fuzzy sets do hold even if the complementation is defined in the way as suggested in this article. In other words, we can express these results obtained with the help of new definition of cardinality of fuzzy sets mathematically in the following manner: 


\section{Proposition 1}

$$
\text { If } \begin{aligned}
A^{c} \subseteq & B^{c} \\
& \text { then } \sum \operatorname{count}\left(A^{c}\right) \subseteq \sum \operatorname{count}\left(B^{c}\right)
\end{aligned}
$$

\section{Proposition 2}

$$
\begin{aligned}
& \sum \operatorname{Count}\left(A^{c}\right)+\sum \operatorname{Count}\left(B^{c}\right)= \\
& \sum \operatorname{Count}\left(A^{c} \cup B^{c}\right)+\sum \operatorname{Count}\left(A^{c} \cap B^{c}\right)
\end{aligned}
$$

\section{Proposition 3}

$$
\begin{aligned}
& \sum \operatorname{count}\left(A^{c} \cap B^{c}\right) \leq \\
& \min \left(\sum \operatorname{count}\left(A^{c}\right), \sum \operatorname{count}\left(B^{c}\right)\right. \\
& \leq \max \left(\sum \operatorname{count}\left(A^{c}\right), \sum \operatorname{count}\left(B^{c}\right)\right) \\
& \sum \operatorname{count}\left(A^{c} \cup B^{c}\right)
\end{aligned}
$$

The differences which are observed in finding cardinality of fuzzy sets using the proposed definition are with the cardinalities of a set, say $\mathrm{A}$ and its union and intersection with its complement $A^{c}$. The reason behind such a claim can be described in the form that in the existing literature, it is believed that fuzzy sets do not obey excluded middle laws. This is one of the most important concepts of fuzzy set theory since the inception of the theory. When it is seen from logical standpoints, it seems that these are due to some shortcomings in the definition of complementation. This is not desirable because there should not be anything common between a set and its complement. But if complement is defined in the proposed manner then it can be seen that those results are based merely on some beliefs without having any logical foundation and hence cannot be taken into consideration for future works. So for future works we should proceed in a way which has some logical foundations.

That is to say that the new definition of complementation would work against the existing literature while yielding the following two results:

$$
A \bigcap A^{c}=\varnothing \text {, the empty set. }
$$

and

$$
A \bigcup A^{c}=\Omega \text {, the universal set. }
$$

Hence it can be said that unlike classical sets fuzzy sets also satisfy excluded middle laws which violate the existing concepts.
We would like to cite a numerical example for illustration purpose.

\section{Numerical Examples}

In this section we have cited an example to show how our definition of cardinality differs from that of the existing ones when the excluded middle laws are taken into considerations.

Let consider the following fuzzy set $\mathrm{A}$ and $A^{c}$ in accordance with reference function

$$
\begin{array}{r}
A=\{(1,0.2,0),(2,0.5,0),(3,0.8,0), \\
(4,1,0),(5,0.7,0),(6,0.3,0)\}
\end{array}
$$

and the complement $A^{c}$ of A would be written in the following manner

$$
\begin{aligned}
A^{c}= & (1,1,0.2),(2,1,0.5),(3,1,0.8), \\
& (4,1,1),(5,1,0.7),(6,1,0.3)\}
\end{aligned}
$$

Then we get

$$
A \bigcup A^{c}=\{(1,1,0),(2,1,0),(3,1,0),(4,1,0),(5,1,0)\}
$$

which is the universal set .

Again we have

$$
\begin{aligned}
& A \bigcap A^{c}=\{(1,0.2,0.2),(2,0.5,0.5),(3,0.8,0.8) \text {, } \\
& (4,1,1),(5,0.7,0.7),(6,0.3,0.3)\}
\end{aligned}
$$

which is the null set.

Hence the cardinalities of the above mentioned sets are

$$
\left|A \cap A^{c}\right|=|\phi| \text {, which is nothing but zero, }
$$

and

$$
\left|A \cup A^{c}\right|=|\Omega| \text {, which is the universal set. }
$$

Here we get cardinalities of the fuzzy sets by using our definition which does not obey the rules established so far. These are the cases where the new definition of complementation produces some results which differ from those of existing beliefs that in case of fuzzy sets A, neither its union with the complement $A^{c}$ is a universal set nor its intersection with the complement $A^{c}$ is a null set. Thus while dealing with cardinalities of these sets, we would have to be contended with a result completely different from the existing results. The suggested definition of cardinality would undoubtedly play an important role in narrowing down the large gap that currently exists in the fuzzy literature. 


\section{Conclusions}

In this article, we intended to revisit the existing definition of cardinality of fuzzy sets and in the process it is found that the cardinality of a fuzzy set especially when dealing with complementation is not defined logically. The reason behind such a claim is contributed to the fact that the existing definition of complementation is not logically defined. We have explained the meaning as well as some motivations. Hence it is obvious that any result which is obtained with the help of something which itself is controversial cannot yield a suitable result. Hence we felt it necessary to define it accordingly. It is observed that the complementation of fuzzy sets defined with the help of reference function seems more logical than the existing one. If this be the case, then there would be problem in finding the cardinality of such a set. It is due to this reason; we would like to propose a new definition of cardinality of fuzzy sets on the basis of membership value. Here efforts have been made to show that if the cardinality is defined with the help of membership value then also it satisfies some propositions which have been established by some researchers. From these perspectives, it can be said that those results which basically found with the help of cardinalities of fuzzy sets can no longer satisfy us. Finally we would like to stress on the fact that since the definition of complementation rooted in reference function discard the fact that fuzzy sets violates excluded middle laws, we would get different cardinalities while dealing with the union and intersection of a set with its complement.

\section{Acknowledgements}

The author would like to thank the anonymous reviewers for their careful reading of this article and for their helpful comments.

\section{References}

[1] Zadeh L A, Inform. and Control, 1965,8: 338-353.

[2] De Luca A, Rermini S, A definition of non probabilistic entropy in the settings of fuzzy set theory, Information and Control, 1972, 20:301-312.

[3] Zazeh L A, A theory of approximate reasoning, Machine Intelligence, 1979, 9: 149-194.

[4] Zazeh L A, A computational approach to fuzzy quantifiers in Natural Languages, Computation and Mathematics, 1983, 9: 149-184

[5] Baruah H K, Fuzzy Membership with respect to a Reference Function, Journal of the Assam Science Society, 1999, 40(.3):65-73.

[6] Baruah H K, Towards Forming A Field of Fuzzy Sets, International Journal of Energy Information and Communications, 2011, 2(1): $16-20$.
[7] Baruah H K, Theory of Fuzzy sets Beliefs and Realities, International Journal of Energy, Information and Communications, 2011, 2(2): 1-22

[8] Baruah H K, In Search of the Root of Fuzziness: The Measure Theoretic Meaning of Partial Presence, Annals of Fuzzy Mathematics and Informatics, 2011, 2(1): $57-68$.

[9] Dhar M, On Hwang and Yang's definition of Entropy of Fuzzy sets, International Journal of Latest Trend Computing, 2011, 2(4): 496-497.

[10] Dhar M, A Note on existing Definition of Fuzzy Entropy, International Journal of Energy Information and Communications, 2012, 3( 1): 1721.

[11] Dhar M, On Separation Index of Fuzzy Sets, International Journal of Mathematical Archives, 2012, .3(3): 932-934.

[12] Dhar M, On Geometrical Representation of Fuzzy Numbers, International Journal of Energy Information and Communications, 2012, 3(2): 2934.

[13] Dhar M, On Fuzzy Measures of Symmetry Breaking of Conditions, Similarity and Comparisons: Non Statistical Information for the Single Patient., Accepted for publication in International Journal of Mathematical Archives, 2012.

[14] Dhar M, A Note on Subsethood measure of fuzzy sets, accepted for publication in International Journal of Energy, Information and Communications, 2012.

[15] Kosko B, Counting with fuzzy sets, Transaction Pattern analysis and Machine Intelligence, IEEE, 1986, 9(4): 556-557

\section{Author's Profiles}

Mamoni Dhar is an Assistant Professor in the department of Mathematics, Science College, Kokrajhar-783370, Assam, India. She received M.Sc degree from Gauhati University, M.Phil degree from Madurai Kamraj University, B.Ed from Gauhati University and PGDIM from Indira Gandhi National Open University. She had received National Scholarship from Matricu lation to Post Graduation. Her research interest is in Fuzzy Set Theory from new perspectives. She has published eleven articles in different national and international journals.

How to cite this paper: Mamoni Dhar,"On Cardinality of Fuzzy Sets", International Journal of Intelligent Systems and Applications(IJISA), vol.5, no.6, pp.47-52, 2013.DOI: 10.5815/ijisa.2013.06.06 